\title{
Photometric and Spectroscopic Study of the $\delta$ Scuti Stars FH Cam, CU CVn and CC Lyn
}

\author{
G. J. Conidis ${ }^{1,2}$, K. D. Gazeas ${ }^{3}$, \\ C. C. Capobianco ${ }^{4}$, W. Ogloza ${ }^{5}$
}

\footnotetext{
${ }^{1}$ Department of Physics and Astronomy, York University, 128 Petrie Science and Engineering Building, 4700 Keele St., Toronto, Ontario, M3J 1P3, Canada

2 David Dunlap Observatory, University of Toronto, P.O. Box 360, Richmond Hill, Ontario, L4C 4Y6, Canada

${ }^{3}$ Department of Astrophysics, Astronomy and Mechanics, Faculty of Physics, University of Athens, GR-15784 Zografos, Athens, Greece

${ }^{4}$ Department of Physics, Engineering Physics and Astronomy at Queen's University, Kingston, ON, K7L 3N6, Canada

${ }^{5}$ Mt. Suhora Observatory of the Pedagogical University, ul. Podchorazych 2, 30-084 Cracow, Poland
}

\begin{abstract}
Three short period ( $\mathrm{P} \ll 1$ day) variable stars from the Hipparcos catalogue targets were observed after suspected misclassification as $\beta$ Lyr eclipsing systems (Perryman et al. 1997), as no secondary component had been noticed in the inspection of their Broadening Functions (BFs) (Rucinski 2002). FH Cam is found to be a multiple star system with a member exhibiting $\delta$ Scuti behaviour. The dominant pulsation frequency is found to be $7.3411 \pm 0.0002 \mathrm{c} / \mathrm{d}$, which corresponds to a pulsation mode of $\ell \leq 1$. We confirmed the pulsations of $\mathrm{CU}$ CVn using photometric observations and found a pulsation frequency of $14.7626 \pm 0.0250 \mathrm{c} / \mathrm{d}$, which is in agreement with the period given in literature. CC Lyn is a non-eclipsing visual binary (CCDM J07359+4302AB), the brighter component $(\mathrm{A})$ is found to be a multi-mode $\delta$ Scuti pulsator, with pulsation frequencies of $5.6402 \pm 0.0004 \mathrm{c} / \mathrm{d}$ and $7.3368 \pm 0.0005 \mathrm{c} / \mathrm{d}$.
\end{abstract}

Accepted: June 7, 2010

Individual Objects: FHCam, CU CVn, CCLyn 


\section{Introduction and Motivation}

Among the contact binary candidates, within the David Dunlap Observatory (DDO) Contact Binary Survey (Rucinski et al. 1999; Pribulla et al. 2009), three candidates FH Cam, CU CVn and CC Lyn (CCDM J07359+4302A) had no sign of a spectroscopic companion. Since these Hipparcos variable stars, have variability periods significantly shorter than half a day, it was conjectured they might be $\delta$ Scuti pulsators (Rucinski 2002).

The purpose of this paper is to confirm the pulsational nature of these variable stars, calculate their pulsational frequencies and, when possible, identify their pulsational harmonic modes. This was performed by using both photometric and spectroscopic observations, which are discussed right after.

\section{Observations and Data Reductions}

For the spectroscopic observations, we used the $1.88 \mathrm{~m}$ Cassegrain telescope at the DDO, which was outfitted with a Thomson Photometrics CCD detector $(1024 \times 1024$ pixels, pixel size $=19.0 \times 19.0 \mu \mathrm{m})$ during the $2000-2001$ observing runs. The Cassegrain spectrograph scale at the dispersion of $10.8 \AA \mathrm{mm}^{-1}$ is about $0.2 \AA_{\text {pixel }}^{-1}$ or about $12 \mathrm{~km} \mathrm{~s}^{-1}$ pixel $^{-1}$. All the spectra collected using the $2000-2001$ configuration were taken at a center wavelength of 5185 $\AA$ (which corresponds to approximately having the $\mathrm{Mg}$ I triplet centered), giving a spectrum coverage of $210 \AA$, on the 1800 lines $\mathrm{mm}^{-1}$ grating. The $2004-$ 2005 observations had been taken with a Jobin-Yvon CCD Camera $(2048 \times 512$ pixels, pixel size $=13.5 \times 13.5 \mu \mathrm{m})$, with a center wavelength of $6440 \AA$, and the same grating. The change in wavelength was due to a suspicion of flexure problems with the spectrograph, so it was decided to select a spectral window which included telluric features from which the flexure of the telescope could be monitored and the residual shift in radial velocity space could be removed for each spectra. It should be noted that spectra collected during March 2005 (five nights) and April 16, 2005 (one night) were badly corrupted by flexure problems from the spectrograph which imposed a random additive constant to the calculated radial velocities after every comparison spectra. All these spectra were discarded and not used in the radial velocity analysis of this paper. However, these aforementioned dates and the number of collected spectra and images are still listed in Table 1, since the photometric data collected in parallel were used for the analysis. During all observing runs, wavelength calibration spectra of an Fe-Ar lamp were taken frequently, depending on the length of the individual exposures. Spectra of multiple template (radial velocity standard) stars, with similar spectral type, were collected at the beginning and the end of each night. The $2004-2005$ observing runs vary in that telluric standards 
were also acquired during the observing run, at the beginning or the end of the night. The spectroscopic data were bias and dark corrected, rectified and wavelength calibrated using the IRAF software package. The procedure of cosmic ray removal was done using a separate, standalone program (Pych 2003). The spectra were then extracted into one-dimensional spectra using IRAF. The radial velocities were calculated using the broadening function (BF) algorithms (Rucinski 1992). The BF method was applied to the entire spectral range of the collected spectra, which has been shown to be a viable method to detect non-radial pulsations (Pribulla et al. 2009). Line profile variation detection was not a possible method of detecting frequencies since the $S / N$ of the spectra were $\sim 12-15$. One should bear in mind that the CCD on the $1.88 \mathrm{~m}$ had been altered multiple times throughout the time spanned by the observations (2000 - 2005), giving an inhomogeneity to the quality of data which induces a slight change in the error bars. This shows up as slightly scattered data taken with center wavelength $6440 \AA$, relative to the data centered at $5185 \AA$.

\begin{tabular}{clccccc}
\hline \hline Date & Star & Images & Spectra (S/N) & Template & Telluric Std. & Obs. \\
\hline $21 / 01 / 2000$ & CU CVn & 0 & $19(15)$ & HD 102870 & N/A & DDO \\
$24 / 04 / 2000$ & FH Cam & 0 & $46(15)$ & HD 48843 & N/A & DDO \\
$24 / 04 / 2000$ & CU CVn & 0 & $12(15)$ & HD 102870 & N/A & DDO \\
$21 / 02 / 2001$ & CC Lyn & 0 & $27(15)$ & HD 32963 & N/A & DDO \\
$22 / 02 / 2001$ & CC Lyn & 0 & $36(15)$ & HD 32963 & N/A & DDO \\
$19 / 12 / 2004$ & CC Lyn & 0495 & $74(13)$ & HD 103095 & HD 87901 & DDO \\
$28 / 12 / 2004$ & CC Lyn & 0273 & $64(13)$ & HD 103095 & HD 87901 & DDO \\
$06 / 03 / 2005$ & CC Lyn & 0024 & $0(0)$ & N/A & N/A & DDO \\
$12 / 03 / 2005$ & CC Lyn & 0078 & $22(12)$ & HD 114579 & HD 87901 & DDO \\
$26 / 03 / 2005$ & CC Lyn & 0071 & $16(12)$ & HD 196821 & HD 87901 & DDO \\
$26 / 03 / 2005$ & CU CVn & 0187 & $13(12)$ & HD 196821 & HD 159139 & DDO \\
$26 / 03 / 2005$ & FH Cam & 0026 & $0(0)$ & N/A & N/A & DDO \\
$09 / 04 / 2005$ & FH Cam & 0177 & $49(12)$ & HD 128167 & HD 136849 & DDO \\
$16 / 04 / 2005$ & FH Cam & 0070 & $29(12)$ & HD 128167 & HD 136849 & DDO \\
$14 / 04 / 2006$ & FH Cam & 0037 & 0 & N/A & N/A & UAO \\
$03 / 05 / 2006$ & FH Cam & 2948 & 0 & N/A & N/A & UAO \\
$06 / 05 / 2006$ & FH Cam & 4758 & 0 & N/A & N/A & UAO \\
$07 / 05 / 2006$ & FH Cam & 4997 & 0 & N/A & N/A & UAO \\
$22 / 05 / 2006$ & FH Cam & 1241 & 0 & N/A & N/A & UAO \\
\hline
\end{tabular}

Table 1: Log of the type and amount of data acquired on a specified evening for a given target at the indicated observatory

The photometric data gathered at the DDO are collected using a $0.15 \mathrm{~m} \mathrm{f} / 8$ refractor, attached in parallel on the $1.88 \mathrm{~m}$ telescope for guiding purposes. This refractor is outfitted with a SBIG ST-6 CCD Camera $(375 \times 241$ pixels, pizel size 
$=8.6 \times 6.5 \mu \mathrm{m}$ ), with a field of view of $15 \times 10$ arcmin. Dark, bias, flat frames were acquired in every observing night. The reduction of the photometric exposures were done with in AIP4WIN (Berry \& Burnell 2000). The CCD exposures were taken without a filter. (Table 2 lists the comparison and check stars for each target-object). Acquiring a check star for $\mathrm{CU} C V \mathrm{n}$ was not possible as the field of view with this setup was too small to allow three bright stars to be simultaneously monitored.

Further observations of FH Cam were carried out from the University of Athens Observatory (UAO), in Athens, Greece with a $0.40 \mathrm{~m} f / 8$ Cassegrain telescope. The observations were collected over 5 nights, in April and May 2006. For the observations obtained on April 14 and May 22, 2006 a SBIG ST-8 CCD detector $(1530 \times 1020$ pixels, pixel size $=9.0 \times 9.0 \mu \mathrm{m})$ was used, giving a field of view of $15 \times 10$ arcmin. For the observations obtained on May 3, 6 and 7, 2006 an ST10-XME CCD detector $(2184 \times 1472$ pixels, pixel size $=$ $6.8 \times 6.8 \mu \mathrm{m})$ was used, giving a field of view of $12 \times 11 \mathrm{arcmin}$. The star was observed in most of the nights for at least 5 hours (typically 5-7 hours), covering more than one period in Bessel B, V, R and I filters. The CCD frames were bias, dark and flat field corrected, using bias, dark and flat images obtained at the beginning or end of each night. A complete list of photometric and spectroscopic observational logs are summarized in Table 1.

\section{Overview of Targets}

Table 2 lists the coordinates, photometric magnitude, spectral classification, the projected stellar-surface equatorial rotation velocity, as well as the comparison and check stars used for the photometric reduction. In addition, it lists the previously published pulsating frequencies and spatial radial velocities for all three targets. FH Cam was classified as a $\beta$ Lyr eclipsing binary system by the light curve obtained by the Hipparcos mission (Perryman et al. 1997). Similarly, CU CVn was initially classified as a $\beta$ Lyr eclipsing binary system by the Hipparcos mission (Perryman et al. 1997), but in 2002 it was discovered to be a $\delta$ Scuti pulsator (Vidal-Sainz J. et al. 2002). CC Lyn (HD 60335) is the brighter component of the visual binary system CCDM J07359+4302AB $\left(m_{A}=6.538, m_{B}=8.257, P A=88^{\circ}, \operatorname{Sep}=2.2^{\prime \prime}\right)$.

\section{Data Analysis}

After converting the dates for the observations to Heliocentric Julian Date (HJD), the photometric data were examined for erroneous outlying points. If data points were found to be outside the $3 \sigma$ amplitude range and showed no temporal-continuity with adjacent points indicated a possible physical origin, 


\begin{tabular}{|c|c|c|c|}
\hline & FH Cam & $\mathrm{CU} C \mathrm{~V}_{\mathrm{n}}$ & CC Lyn \\
\hline R.A. & 075739.7509 & 134820.1169 & 073555.9777 \\
\hline Dec. & +773435.121 & +312403.801 & +430151.453 \\
\hline$m_{v}$ & 6.903 & 7.537 & 6.538 \\
\hline Sp. Class & A8V & A7V & F0-2V \\
\hline$v \sin i(\mathrm{~km} / \mathrm{s})$ & 45 & 155 & $\leq 15$ \\
\hline $\begin{array}{l}\text { Comparison } \\
\text { star }\end{array}$ & $\begin{array}{l}\text { GSC 4531-1441 } \\
(\text { HD 63311) }\end{array}$ & $\begin{array}{c}\text { GSC 2537-0397 } \\
\text { (HD 120279) }\end{array}$ & GSC 2966-0661 \\
\hline $\begin{array}{l}\text { Check } \\
\text { star }\end{array}$ & $\begin{array}{l}\text { GSC 4543-1724 } \\
\text { (HD 63855) }\end{array}$ & $N / A$ & GSC 2966-0781 \\
\hline$\nu_{\text {pulse }}(\mathrm{c} / \mathrm{d})$ & $3.67002(1)(\mathrm{HIP})$ & $\begin{array}{c}7.3709(8)(\mathrm{HIP}) \\
14.7418(6) \dagger \\
12.5612(3) \dagger\end{array}$ & $2.81990(4)(\mathrm{HIP})$ \\
\hline $\mathrm{RV}_{\circ}(\mathrm{km} / \mathrm{s})$ & $\begin{array}{c}3.0 \\
4.5 \pm 7.1 \dagger \dagger\end{array}$ & -4.1 & $\begin{array}{c}\text { Variable } \\
20.6 \pm 2.0 \ddagger\end{array}$ \\
\hline
\end{tabular}

Table 2: Overview of basic information of the three observed targets. The Comparison and Check stars were used for the photometric reduction. Spectral types, vsini and radial velocities were taken from Pribulla et al. 2009 or otherwise mentioned. Previously published pulsating (or orbital) frequencies and spatial radial velocities of targets are taken from Hipparcos catalog (pulsating frequencies are double the orbital frequency mentioned by HIP) and $\dagger$ Vidal-Sainz J. et al 2002, †† Grenier et al. 1999, $\ddagger$ Wilson 1953

the points were discarded. The remaining time series was then analyzed using the software package Period04 (Lenz \& Breger 2005). This package was used to calculate the Discrete Fourier Transforms of the data, enabling a determination of the oscillation frequencies. The errors were calculated using the Monte Carlo error estimation package within Period04, where 1000 iterations were used for all the photometric data. The signal to noise ratio $(S / N)$ was also calculated by Period04. The resulting frequencies and their signal to noise ratio are both listed in Table 3. The amplitude spectra of all stars are presented for each frequency in Figure 5. In order to attain the phase plots for the photometric data, the location of the photometric light curve minima of the pulsations $T_{\text {min }}$ was identified. In the case of FH Cam, the B filter photometry was used to identify $T_{\min }$ and was used to create all phase plots in the $\mathrm{V}, \mathrm{R}$ and I filters. In the cases of CU CVn and CC Lyn, the unfiltered photometry was used to determine $T_{\text {min }}$. 
In the case of the photometric data taken from the UAO, the data was of very good quality ( $\sigma_{\text {mag }} \approx 1 \mathrm{mmag}$ ) and was acquired in the $\mathrm{B}, \mathrm{V}, \mathrm{R}$, I Bessel passband filters. This enabled the use of the software package FAMIAS (Zima 2008), which requires multi-passband filtered data to identify the spherical harmonics associated with detected frequencies.

\begin{tabular}{|c|c|c|c|c|}
\hline \multicolumn{5}{|l|}{ Photometric } \\
\hline Star & $\begin{array}{l}\nu_{\text {pulse }} \\
\text { (c/d) }\end{array}$ & $\begin{array}{c}\text { Amplitude } \\
\text { (mag) }\end{array}$ & $\mathrm{N}$ & $\mathrm{S} / \mathrm{N}$ \\
\hline$\overline{\mathrm{FH} \mathrm{Cam}}{ }^{\dagger}$ & $07.3411 \pm 0.0002$ & $0.0313 \pm 0.0003$ & 3808 & 4.60 \\
\hline $\mathrm{CU} C \mathrm{~V}_{\mathrm{n}}$ & $14.7626 \pm 0.0250$ & $0.0300 \pm 0.0010$ & 0284 & 4.81 \\
\hline CC Lyn $\nu_{1}^{*}$ & $05.6402 \pm 0.0004$ & $0.0245 \pm 0.0010$ & 0776 & 5.49 \\
\hline CC Lyn $\nu_{2}^{*}$ & $07.3368 \pm 0.0005$ & $0.0123 \pm 0.0010$ & 0776 & 3.38 \\
\hline \multicolumn{5}{|c|}{ Spectroscopic } \\
\hline Date & $\begin{array}{c}\mathrm{RV}_{\circ} \\
(\mathrm{km} / \mathrm{s})\end{array}$ & $\begin{array}{c}\text { Amplitude } \\
(\mathrm{km} / \mathrm{s})\end{array}$ & $\mathrm{N}$ & \\
\hline \multicolumn{5}{|l|}{ FH Cam } \\
\hline $24 / 04 / 2000$ & $+03.21 \pm 0.17$ & $2.78 \pm 0.14$ & 0050 & \\
\hline $09 / 04 / 2005$ & $-06.88 \pm 0.10$ & $1.64 \pm 0.24$ & 0049 & \\
\hline \multicolumn{5}{|l|}{$\mathrm{CU} C \mathrm{CV}_{\mathrm{n}}$} \\
\hline All Spectra & $-00.93 \pm 0.17$ & $1.49 \pm 0.42$ & 0032 & \\
\hline \multicolumn{5}{|l|}{ CC Lyn } \\
\hline $21 / 02 / 2001$ & $+17.09 \pm 0.16$ & $2.52 \pm 0.20$ & 0027 & \\
\hline $22 / 02 / 2001$ & $+12.83 \pm 0.12$ & $1.94 \pm 0.19$ & 0036 & \\
\hline $19 / 12 / 2004$ & $+33.87 \pm 0.28$ & $3.11 \pm 0.38$ & 0074 & \\
\hline $28 / 12 / 2004$ & $+51.30 \pm 0.23$ & $6.25 \pm 0.32$ & 0064 & \\
\hline
\end{tabular}

Table 3: A listing of the pulsating frequencies and their corresponding amplitudes, found in our analysis, as well as the associated velocity amplitudes and phases found from folding the RV data to the same periods. $\mathrm{N}$ is the number of exposures used to calculate the period listed. ${ }^{\dagger}$ The photometric data used for FH Cam was acquired through a B filter, while the remaining two stars had their photometric data collected through clear apertures.

Similarly, the radial velocity data was converted to HJD and phase plots where generated using the photometrically determined frequencies for each star. The value $T_{\min }$ used for the radial velocity phase plot was the same used for 
the corresponding stars photometric plot. The phased radial velocity data was then fitted with a sinusoidal curve, to determine the amplitude of the radial velocity variations, and the radial velocity offset $\left(\mathrm{RV}_{\circ}\right)$ (Table 3 ).

\subsection{FH Cam}

From the analysis of the $\mathrm{B}$ filter photometric data, the dominant period was determined to be $7.3411 \pm 0.0002 \mathrm{c} / \mathrm{d}$ (see Figure 5), which is in agreement with double the Hipparcos frequency $(7.34004 \pm 0.00002 \mathrm{c} / \mathrm{d})$. The amplitude of the photometric oscillation decreases as the filter's transparent window moves toward longer wavelengths, which supports the classification of $\mathrm{FH} \mathrm{Cam} \mathrm{being}$ a pulsator and not an eclipsing binary (See Figure 1 and Table 4).

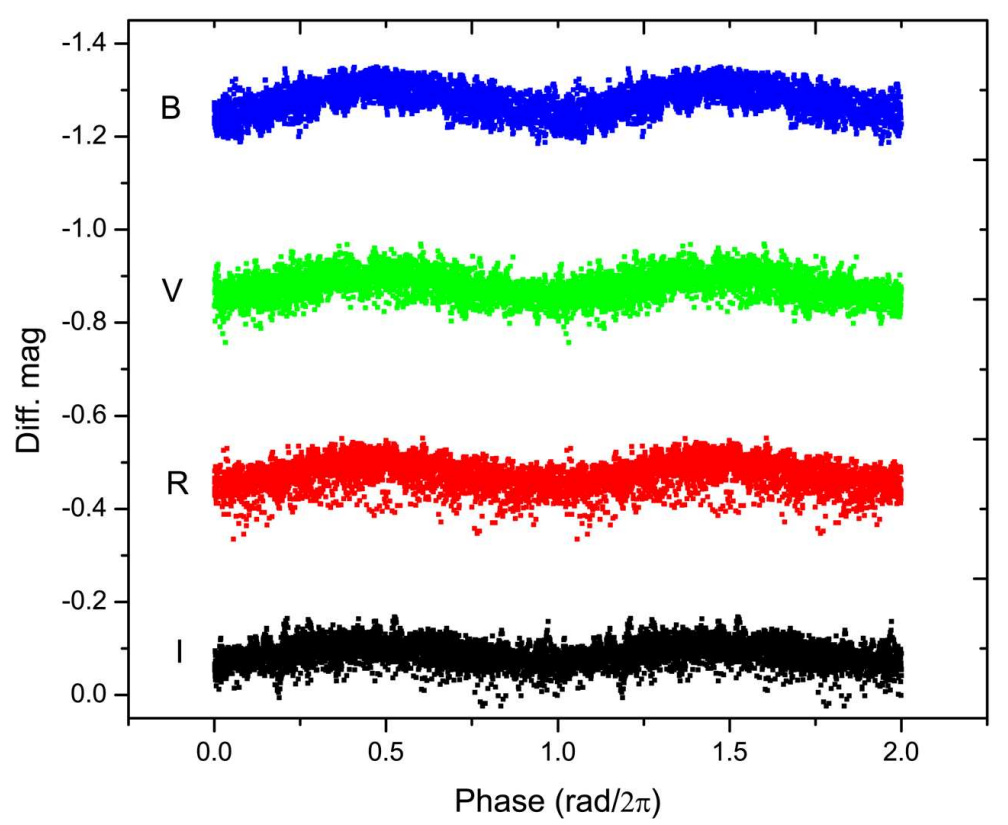

Figure 1: Bessel passband photometry of FH Cam. The decrease in the amplitude of the oscillation, in the order of $B, V, R, I$ passband filters (Top to Bottom) clearly indicates a pulsation is the cause of the change in magnitude. The pulsation shown has a frequency corresponding to $7.3411 \mathrm{c} / \mathrm{d}$. 


\begin{tabular}{lcc}
\hline \hline Filter & $\begin{array}{c}\text { Amplitude } \\
(\mathrm{mmag})\end{array}$ & $\begin{array}{c}\text { Phase } \\
\left(\mathrm{rad}(2 \pi)^{-1}\right)\end{array}$ \\
\hline $\mathrm{B}$ & $30.97 \pm 0.37$ & $0.762 \pm 0.002$ \\
V & $23.73 \pm 0.37$ & $0.717 \pm 0.003$ \\
$\mathrm{R}$ & $22.87 \pm 0.42$ & $0.715 \pm 0.003$ \\
I & $17.63 \pm 0.44$ & $0.697 \pm 0.005$ \\
\hline
\end{tabular}

Table 4: The amplitude and phase from fitting a sinusoidal function to the data in Figure 1.

The software package FAMIAS was used to calculate models simulating the frequencies found from the Bessel $B, V, R$, I photometry in order to constrain the radial order $\ell$. The assumptions regarding the models of $\mathrm{FH} \mathrm{Cam}$ are the following: $M=2.0 M_{\odot}, T_{\text {eff }}=7650 \pm 100 \mathrm{~K}$ and $\log (g)=4.0 \pm 0.1$, which are the average values of 10 stars with a spectral classification of A8V. A grid within the Kurucz atmospheric models were found to fit the data, with a range of metallicities of $0<\mathrm{Z}<0.02$ and micro turbulence ranging $0 \mathrm{~km} / \mathrm{s}<\xi<4$ $\mathrm{km} / \mathrm{s}$ with no noticeable change to the values of $\ell$ outputted by the software package. The modal orders $\ell=0$ to 5 were found to agree for all the amplitude ratio vs. phase difference plots except the $\mathrm{R}$ and $\mathrm{V}$ amplitude ratio vs. phase diagram (see Figure 6) which shows that only the $\ell=0,1$ radial pulsations are allowed. Hence, we find that the detected pulsation must have an order of $\ell \leq$ 1 .

The systemic velocity $\left(\mathrm{RV}_{\circ}\right)$ and the amplitude of the pulsation, are variable on a nightly basis (See Table 3 and Figure 2), which indicates that FH Cam is a multiple system with a non-spectroscopic and non-visual companion. While the time-dependent amplitudes of the radial velocity pulsations are most likely the result of other excited (low amplitude) pulsation frequencies constructively or destructively interfering with the observed pulsation.

\section{2. $\mathrm{CU} \mathrm{CVn}$}

From the analysis of the unfiltered photometric data, the dominant frequency was determined to be $14.7626 \pm 0.0250 \mathrm{c} / \mathrm{d}$ (see Figure 5), which is in complete agreement with the spectroscopic data (See Figure 3), the dominant frequency found by Vidal-Sainz et al. $2002(14.7418 \pm 0.00004 \mathrm{c} / \mathrm{d})$, and the doublefrequency from Hipparcos $\left(14.7418 \pm 0.00001 \mathrm{c} / \mathrm{d}^{1}\right)$.

\footnotetext{
${ }^{1}$ Hipparcos assumed $\mathrm{CU} C V \mathrm{n}$ was a binary, so multiplication by two is needed to obtain the correct frequency.
} 

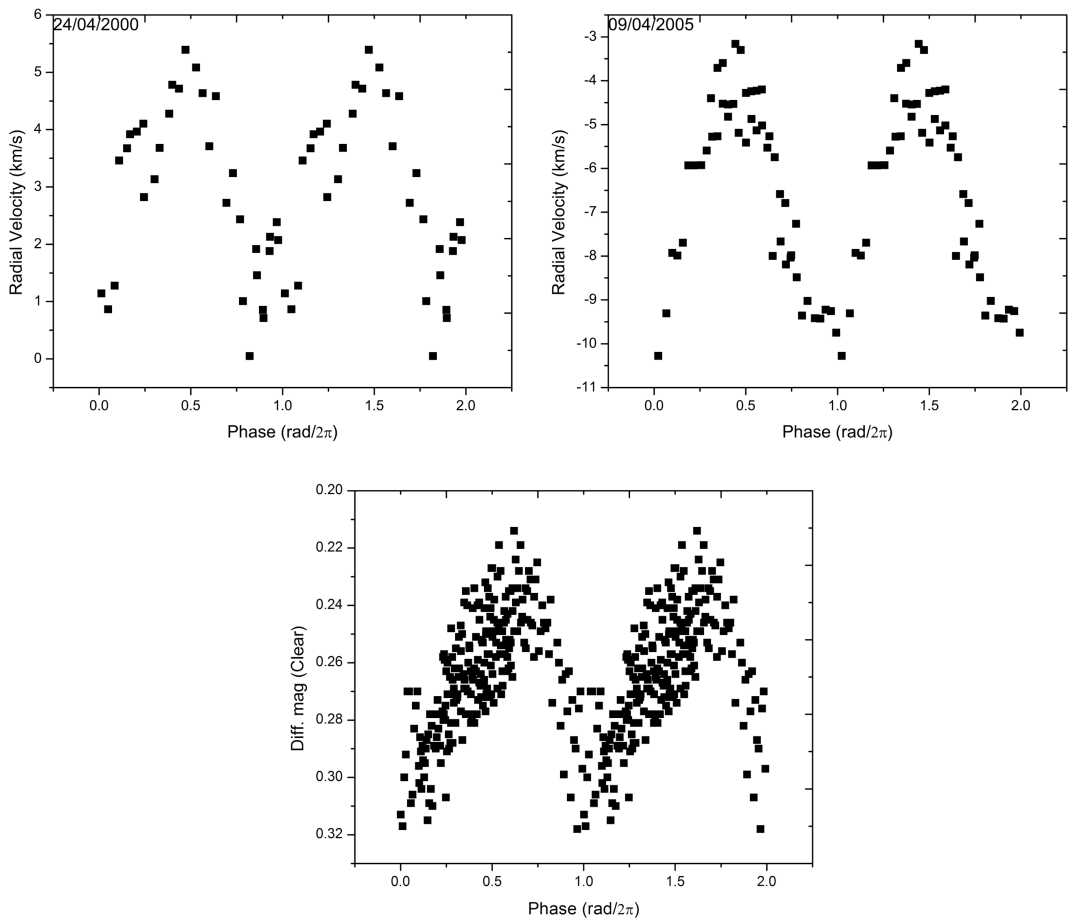

Figure 2: Radial velocity and differential magnitude phase plots for FH Cam, for the data acquired at the DDO. The frequency of pulsation is $7.3411 \mathrm{c} / \mathrm{d}$.

The systemic velocity was found to be $-0.93 \pm 0.17 \mathrm{~km} / \mathrm{s}$ for all the spectra acquired during the months of January to March in the year 2000.

\subsection{Lyn}

From the analysis of the unfiltered photometric data (See Figure 4), the dominant frequency was determined to be $5.6402 \pm 0.0004 \mathrm{c} / \mathrm{d}$ (see Figure 5), which is also in agreement with the double-frequency from Hipparcos (5.63980 \pm 0.00002 days). This is also in strong agreement with the radial velocity data being folded about this frequency (Figure 4). Interestingly, the ratio of the dominant (largest amplitude) frequency $\left(\nu_{1}^{*}\right)$ to the second dominant frequency $\left(\nu_{2}^{*}\right)$ gives a value of $0.768 \pm 0.005$, which is in agreement with the value 0.77 for the ratio of first radial overtone pulsation to the fundamental 

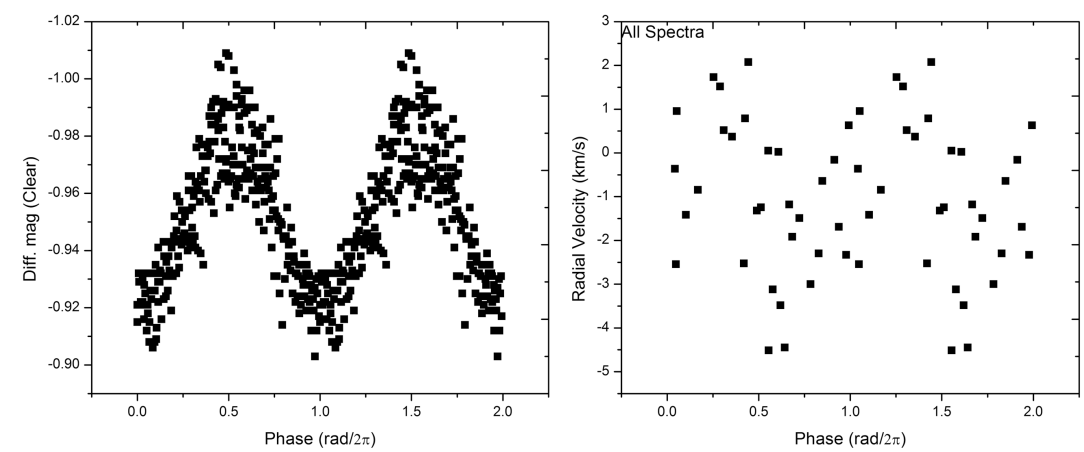

Figure 3: Radial velocity and differential magnitude phase plots for CU CVn for data acquired at the DDO, for the found frequency of $14.7626 \mathrm{c} / \mathrm{d}$.

radial mode pulsation (Kurtz 2006). The value of this ratio could be an indication that CC Lyn is a Pop I star (Breger 2000), which has a frequency ratio of $0.769 \pm 0.001$. The aforementioned agreement with the expected ratio for a double-mode $\delta$ scuti pulsator must be confirmed through mode id calculations to properly secure its classification as a double-mode pulsator.

CC Lyn also shows variations in its systemic radial velocity and radial velocity pulsation amplitude and phase, as indicated in Table 3. CC Lyn is known to be in a binary system, which we confirmed through the observed change in the systemic velocity in each observing run. The variational change in amplitude and phase is expected in a multi-mode pulsator, as the different pulsations will beat against each other creating constructive and destructive interference, observed as a modulating pulsation. 

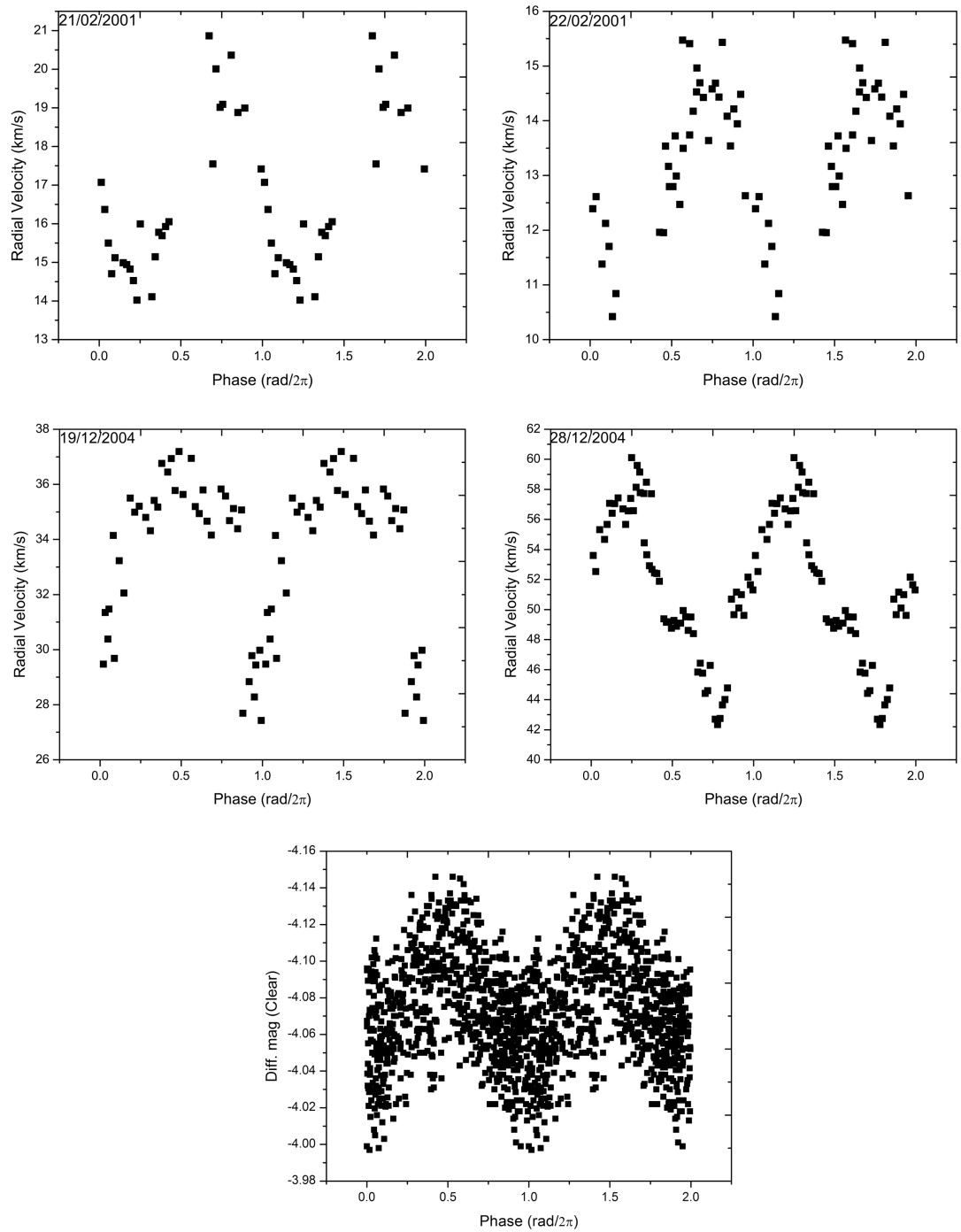

Figure 4: Radial velocity and differential magnitude phase plots for CC Lyn, for the frequency of $5.6402 \mathrm{c} / \mathrm{d}$. 
34 Photometric and Spectroscopic Study of FH Cam, CU CVn and CC Lyn
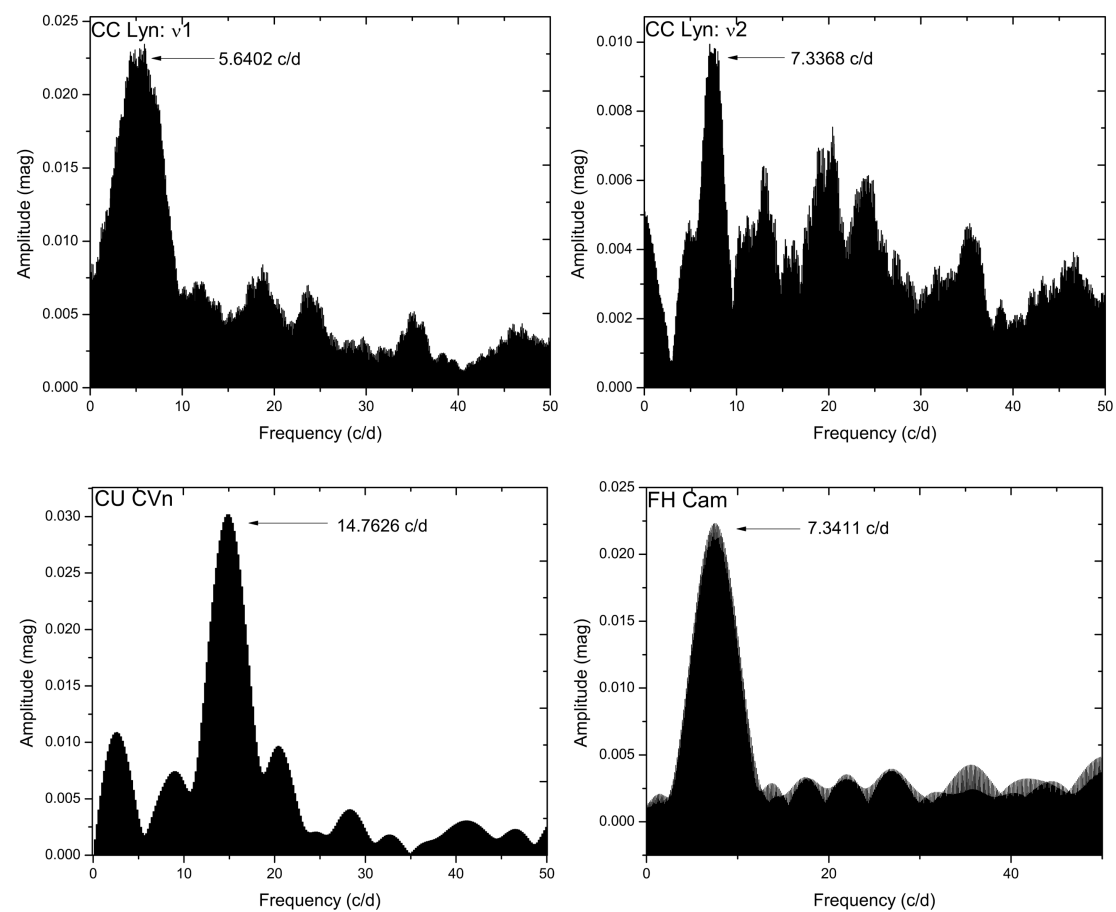

Figure 5: The amplitude spectra of CC Lyn, CU CVn, \& FH Cam. 

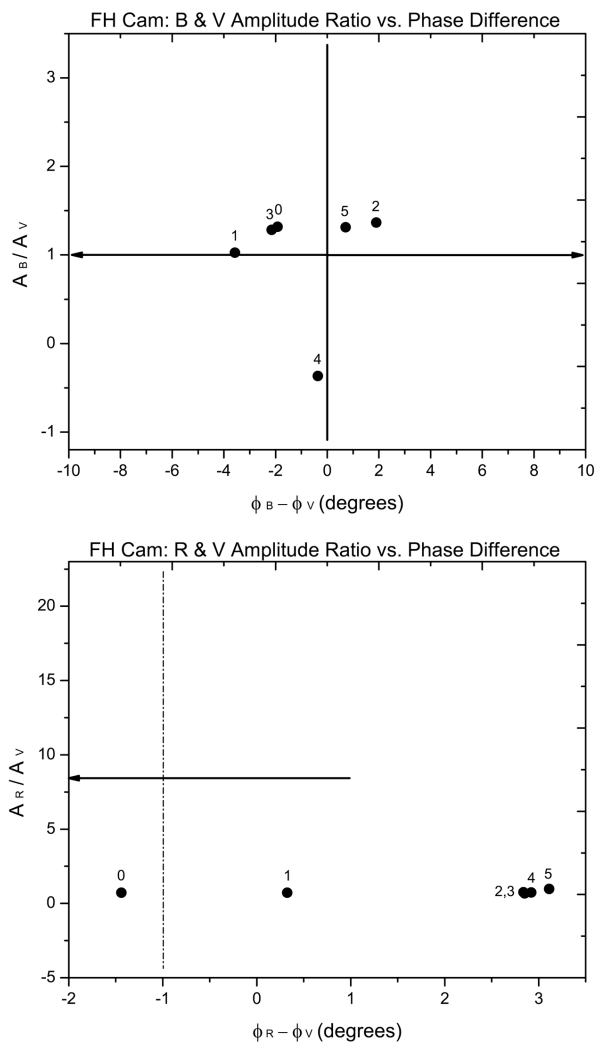

Figure 6: The Amplitude ratios vs. Phase differences for FH Cam are shown, for the B-V and R-V band photometry. The modal orders are indicated by the number print above a given data point. It can be seen that $\mathrm{FH}$ Cam must have a radial pulsation modal order, $\ell \leq 1$. The error values are indicated by the size of the dot used to indicate the appropriate modal order. The dotted vertical axis in the $\mathrm{R}-\mathrm{V}$ diagram indicates that the vertical axis has been displaced from intersecting the origin so it can appear in the plot (origin is at $\sim(-106,8)$ ). The arrow heads indicate the measured phase difference was larger than plotted in the indicated direction: $B-V$ phase $=(-160,160)$, V-R phase $=(-215,1)$ degrees. 


\section{Conclusion}

We clearly see that all stars analyzed have the correct spectral type to be included in the instability strip for $\delta$ Scuti stars (Breger 2000), and have shown pulsations with $\nu>5 \mathrm{c} / \mathrm{d}$. These results securely place FH Cam, CU CVn, and CC Lyn in the category of $\delta$ Scuti pulsators.

FH Cam is shown to have only one detectable pulsation, which is found to have $\ell \leq 1$. There is also a decrease in amplitude of the light curves as the filter's pass-band is centered at longer wavelengths, further supporting the claim that FH Cam is a $\delta$ Scuti pulsator. The variability in the systemic radial velocity also indicates $\mathrm{FH}$ Cam is part of a multiple system.

$\mathrm{CU} \mathrm{CVn}$ is observed to have variability in both the photometric and spectroscopic data sets, implying we have detected a pulsation, with a frequency of $14.7626 \pm 0.0250 \mathrm{c} / \mathrm{d}$. Hence, the observed high frequency in conjunction with the spectral type being $\mathrm{FO}-2 \mathrm{~V}$ confirms that $\mathrm{CU} C \mathrm{Cn}$ is a $\delta$ Scuti star. There was no noticeable variability of $\mathrm{RV}$ 。 for this object, but a longer baseline of data will be needed to rule out binarity.

We have found CC Lyn to have two detectable pulsations. The ratio of these two periods might indicate the fundamental and first overtone radial pulsations, but it is not quite definite. The change in systemic radial velocity confirms the binarity of CC Lyn.

Acknowledgments. The authors would like to express their appreciation to Prof. Slavek M. Rucinski for his support during the initiation of the project, and his guidance and invaluable discussions throughout all stages of the present paper. We also would like to express our gratitude to the telescope operators at the David Dunlap Observatory, Heidi DeBond, Wen Lu, and Jim Thompson, for their assistance during the observing runs and to Prof. Marshall L. McCall for his constructive advice. We finally express our gratitude to the anonymous referee for his insightful advice and comments regarding our work.

\section{References}

Berry, R., \& Burnell, J., The Handbook of Astronomical Image Processing, Willmann-Bell, Richmond (Virginia), 2000

Breger, M. 2000, ASPC, 210, 3B

Grenier, S., Baylac, M.O., Rolland, L., et al. 1999, A\&AS, 137, 451G

Kurtz, D. W. 2006, CoAst, 147, 6

Lenz, P. \& Breger, M. 2005, CoAst, 146, 53

Perryman, M. A. C., Lindegren, L., Kovalevsky, J., et al. 1997, A\&A, 323, 49

Pych, W. 2003, PASP, submitted (arXiv:astro-ph/0311290v1) 
Pribulla, T., Rucinski, S. M., Blake, R. M., et al. 2009, AJ, 137, 3655

Rucinski, S. M. 1992, AJ, 104, 1968

Rucinski, S. M. 2002, PASP, $114,1124 \mathrm{R}$

Rucinski, S., Lu, W. 1999, JRASC, 93, 186R

Vidal-Sáinz, J., Gomez-Forrellad, J. M., García-Melendo, E., Wils, P., \& Lampens, P. 2002, IBVS, 5331, 1

Wilson, R.E. 1953,General Catalogue of Stellar Radial Velocities, Carnegie Institution of Washington Publ. 601, Washington D.C

Zima, W. 2008, CoAst, 155, 17 
